WPSReview International on Sustainable

Housing and Urban Renewal (RI-SHUR)

\title{
CONDUCCIÓN SOCIAL COOPERATIVA \\ EN CIUDADES INTELIGENTES
}

\author{
Jamal Toitouh El Alamin \\ Universidad de Málaga
}

Artículo Recibido: 28/04/2018

Artículo Aceptado: 14/09/2018

\section{Resumen}

Uno de los mayores retos a los que se enfrentan las sociedades modernas es la movilidad urbana. La congestión de las carreteras y los problemas de seguridad vial son considerados factores clave en la degradación de la calidad de vida de los ciudadanos. Las innovaciones tecnológicas que consigan mejorar la movilidad marcarán gran parte del futuro de las ciudades inteligentes. Una de las tecnologías más prometedoras aplicadas a este ámbito son las redes vehiculares ad hoc. Estas redes de comunicación inalámbrica permiten la comunicación directa, gratuita y dinámica entre vehículos cercanos y de estos con elementos de la infraestructura vial.

Estas redes de comunicación están marcando la industria del automóvil actual, ya que han propiciado el surgimiento del vehículo conectado y la Conducción Social Cooperativa (Cooperative Social Driving, CSD). El acceso a distintas fuentes de información permite ampliar enormemente las funcionalidades del vehículo. EI CSD permite que los vehículos cercanos puedan interactuar intercambiando información relevante en tiempo real. Esta información puede haber sido recogida por los sensores del vehículo, introducida por usuarios o recibida de otro vehículo. De este modo los conductores circulan con una percepción más completa del estado real del tráfico y pueden ser advertidos sobre la existencia de obstáculos, accidentes, peatones en la vía u otro cualquier hecho importante. También se pueden desarrollar utilidades, como crear nubes para el intercambio de documentos, fotos, vídeos y juegos.

El CSD se sustenta en el intercambio de archivos, siendo capital que la transferencia de los mismos sea óptima. El que un mensaje llegue y que lo haga a tiempo representa la diferencia de evitar o no una situación peligrosa. Nuestro grupo de investigación ha desarrollado protocolos para este fin, y ha optimizado los existentes 


\title{
WPSReview International on Sustainable
}

\author{
Housing and Urban Renewal (RI-SHUR)
}

para que comiencen a ser útiles en situaciones reales de velocidades variadas y volatilidad de la conexión.

Por lo general, son expertos quienes por su experiencia o llevando a cabo experimentos de laboratorio proponen la configuración de un protocolo. En el presente artículo se presenta la aplicación de la Inteligencia Computacional para la configuración automática e inteligente de protocolos aplicados en redes vehiculares. Para ello se ha definido la configuración de protocolos como un problema de optimización y se han diseñado algoritmos inspirados en la naturaleza para resolverlo. Entre los algoritmos empleados destacan los Algoritmos Genéticos, que se basan en la Teoría de Darwin de la evolución de las especies, y los algoritmos de Optimización por Cúmulo de Partículas, inspirados en el comportamiento social de los animales como las aves en una bandada.

Estos algoritmos se han combinado con un simulador de redes vehiculares realista y de forma automática han calculado configuraciones óptimas que han mejorado considerablemente el rendimiento de los protocolos de comunicación analizados. En las pruebas reales en ciudad (Málaga), sólo los protocolos optimizados empleando nuestra metodología han podido transmitir la información que se requería de un vehículo a otro. Las aplicaciones posibles son innumerables, y la tecnología está lista para generar productos que se acepten en el mercado actual.

\section{Abstract}

Most of the world population lives in urban areas, and it is expected that the number of inhabitants in cities will be $75 \%$ of the world's population by 2050 . Thus, a wide range of challenges have to be faced by the different city stakeholders in order to mitigate the negative effects of a very fast growth of such urban areas. One of the main concerns in modern cities is mobility. The vast increment in the volume of urban road traffic experienced during the last decades causes serious issues that have be confronted with new tools. Traffic jams bother the daily life of the population, mainly because traffic congestion causes longer trip times and a larger associated pollution, not to mention the economic losses due to delays and other transport problems. Thus, great efforts are being made to develop Smart Mobility solutions.

Vehicular ad hoc networks (VANETs) are based on the spontaneous creation of a wireless network for data exchange between vehicles, mainly. Wireless networking technologies are used in VANETs, including short range radio and cellular. These communication networks are leading the automotive industry today, as they have led to the emergence of the connected vehicle and the Social Cooperative Driving (CSD). CSD allow drivers and vehicles to access different data sources in order to increase the knowledge about the current road traffic status. Besides, other types of infotainment services and applications can be deployed using VANETs.

File transferring is always an essential service in VANETs since their ultimate purpose is the exchange of information among the nodes. Thus, it is desirable to optimize the

WPS RI-SHUR, nำ-8, 2018, vol.1, ISSN: 2387-1768 


\section{WPSReview International on Sustainable \\ Housing and Urban Renewal (RI-SHUR)}

file transferring protocol to provide the applications with the best service possible. One of the ways to get such an efficient protocols is to better fit their configuration parameters to the VANETs specifications. This study proposes the application of Computational Intelligence to obtain automatically optimal protocol configurations for vehicular environments. The main idea is to define the protocol configuration as an optimization problem and address it by using intelligent tools, such as metaheuristics, and a realistic simulation procedure to evaluate the obtained solutions.

In this work, we have carried out a real world testbed at streets of Málaga in order to study the performance of the analyzed file transfer protocol by exchanging information between cars in a real urban. These experiments have confirmed the efficiency of configuring VANET nodes with protocols improved by applying our methodology.

\section{Palabras clave}

movilidad inteligente; redes vehiculares ad hoc; conducción social cooperativa; inteligencia computacional; Actuaciones sostenibles del espacio urbano.

\section{Key words}

smart mobility, Vehicular ad hoc networks; Social Cooperative Driving; Computational Intelligence; sustainability actions in urban places

\section{1.- Introducción}

Desde que las diferentes civilizaciones empezaron a organizarse en núcleos urbanos se pudo comprobar que la movilidad, tanto interna como inter-urbana, condicionaría el desarrollo de las ciudades. En la actualidad, con la mayoría de la población mundial viviendo en ciudades (se espera que en 2050 sobre un $70 \%$ de la población mundial se concentre en núcleos urbanos), la movilidad es uno de los grandes retos a los que nos tenemos que enfrentar. Según estimaciones de la Comisión Europea los problemas de congestión de las redes viales cuestan un punto porcentual del Producto Interior Bruto de los presupuestos de la Unión, lo que se traduce en un sobrecoste del orden de mil millones de euros al año. A su vez, se estima que el $23 \%$ de la polución que existe en las ciudades europeas lo generan los vehículos de transporte por carretera y a todo esto hay que añadirle los costes humanos y económicos de los accidentes que se producen en las redes viales. Así, desde diferentes iniciativas de Ciudades Inteligentes o Smart Cities se están invirtiendo innumerables esfuerzos en proveer de sistemas de Movilidad Inteligente o Smart Mobility para ofrecer una respuesta adecuada al reto de proporcionar un transporte urbano más eficiente, seguro y cómodo, que está siendo cada vez más exigente.

Gracias a la aplicación de los últimos avances en tecnologías de la información y la comunicación (TIC) en entornos de movilidad vial se ha desarrollado la aparición de las redes vehiculares ad hoc o VANET (vehicular ad hoc networks). 


\title{
WPSReview International on Sustainable
}

\author{
Housing and Urban Renewal (RI-SHUR)
}

Estas redes de comunicación inalámbrica sin infraestructura se forman de forma espontánea, principalmente entre vehículos cercanos y distintos elementos de la infraestructura vial como semáforos, señales, paneles o sensores. Estas redes de comunicación inalámbrica permiten la comunicación directa, gratuita y dinámica entre vehículos cercanos y de estos con elementos de la infraestructura vial (Campolo y col., 2015)

Las VANET están marcando la industria del automóvil actual, ya que han propiciado el surgimiento del vehículo conectado y la Conducción Social Cooperativa (Cooperative Social Driving, CSD). El acceso a distintas fuentes de información permite ampliar enormemente las funcionalidades del vehículo. El CSD permite que los vehículos cercanos puedan interactuar intercambiando información relevante en tiempo real. Esta información puede haber sido recogida por los sensores del vehículo, introducida por usuarios o recibida de otro vehículo. De este modo los conductores circulan con una percepción más completa del estado real del tráfico y pueden ser advertidos sobre la existencia de obstáculos, accidentes, peatones en la vía u otro cualquier hecho importante. También se pueden desarrollar utilidades, como crear nubes para el intercambio de documentos, fotos, vídeos y juegos.

Aplicando CSD se pueden desarrollar aplicaciones revolucionarias en el ámbito de la seguridad y la eficiencia vial, siendo un eje fundamental de los futuros Sistemas Inteligentes de Transporte o Intellingent Transportation Systems (ITS). Dichas aplicaciones facilitarán que los vehículos puedan intercambiar información avisando sobre futuras maniobras o situaciones peligrosas, de este modo se podrá asistir al conductor para evitar situaciones de peligro e incluso accidentes, lo cual es importante porque se estima que el $90 \%$ de los accidentes tienen como causa principal el error humano. A su vez, se desarrollarán aplicaciones que mejorarán la eficiencia de los transportes por carretera proponiendo rutas e itinerarios acordes con el estado del tráfico en tiempo real y los perfiles de cada conductor.

La Figura 1 muestra dos casos de aplicación de CSD para proporcionar movilidad inteligente en nuestras ciudades: a) aviso de accidente de tráfico en la vía pública, reduzca la velocidad y/o cambie de ruta; y b) precaución porque hay un vehículo de emergencias acercándose a su posición. 


\section{WPSReview International on Sustainable}

\section{Housing and Urban Renewal (RI-SHUR)}

Figura 1. Casos de uso de la CSD (C2C-CC, 2015)

\section{a) Aviso de accidente de tráfico en la vía pública}

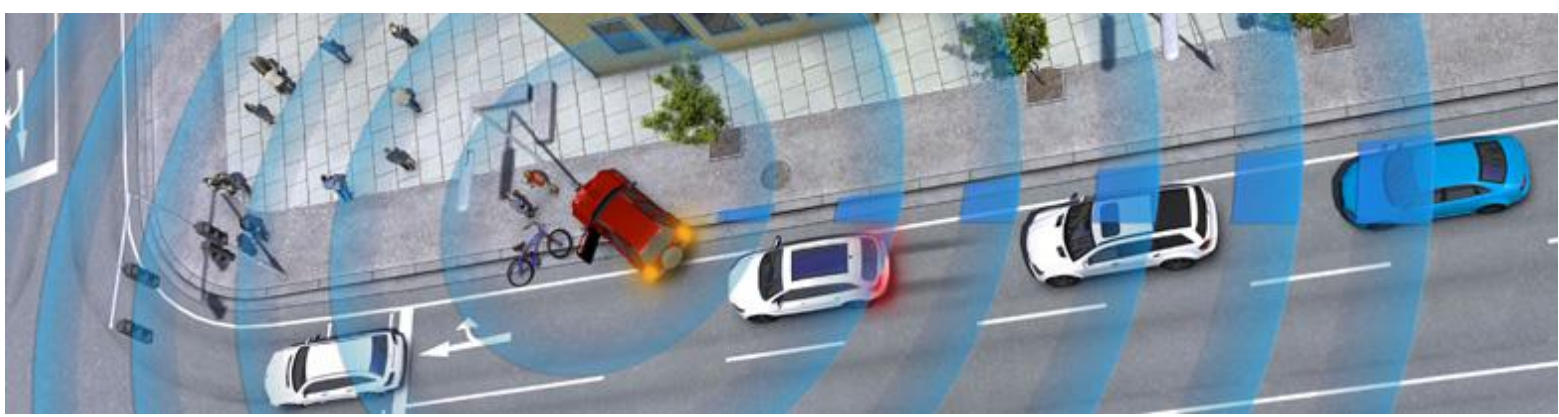

b) Vehículo de emergencias acercándose a su posición

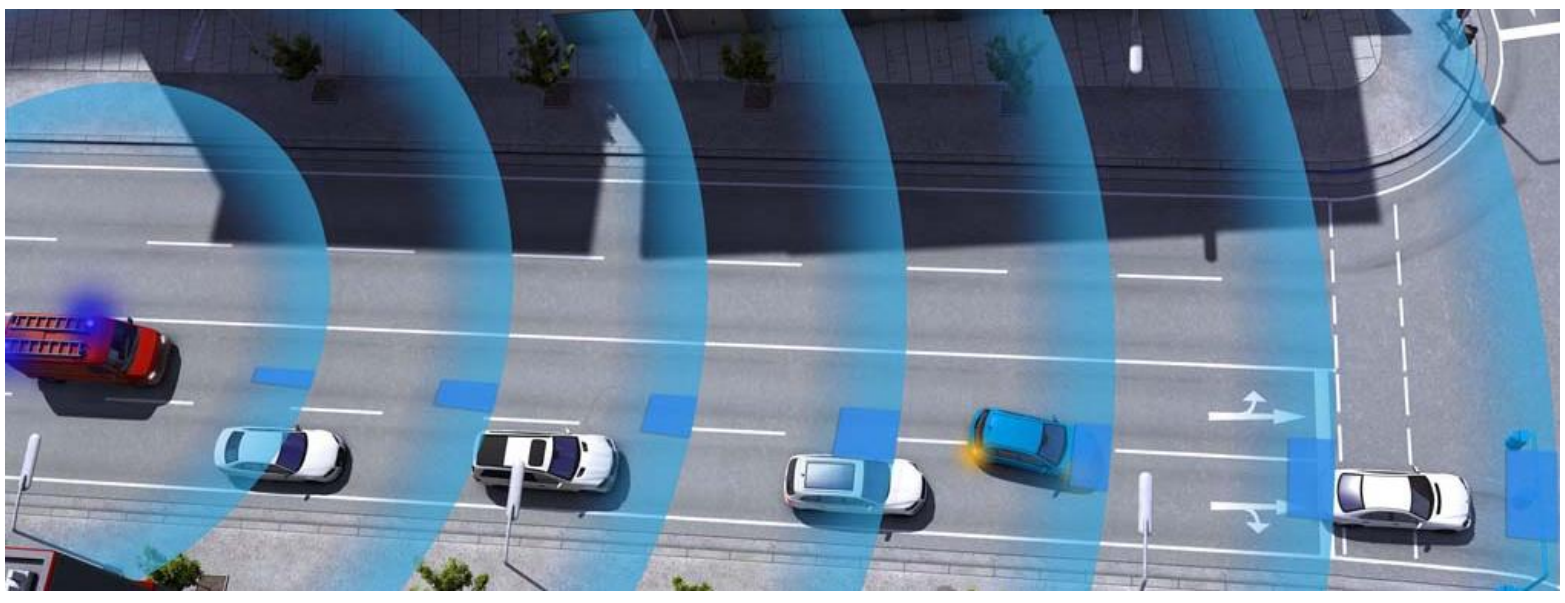

EI CSD se sustenta en el intercambio de archivos, siendo capital que la transferencia de los mismos sea óptima. El que un mensaje llegue y que lo haga a tiempo representa la diferencia de evitar o no una situación peligrosa. Nuestro grupo de investigación ha desarrollado protocolos para este fin, y ha optimizado los existentes para que comiencen a ser útiles en situaciones reales de velocidades variadas y volatilidad de la conexión (CARLINK, 2006). 


\title{
WPSReview International on Sustainable
}

\author{
Housing and Urban Renewal (RI-SHUR)
}

Por lo general, son expertos quienes por su experiencia o llevando a cabo experimentos de laboratorio proponen la configuración de un protocolo. En el presente artículo se presenta la aplicación de la Inteligencia Computacional para la configuración automática e inteligente de protocolos aplicados en redes vehiculares. Para ello se ha definido la configuración de protocolos como un problema de optimización y se han diseñado algoritmos inspirados en la naturaleza (metaheurísticas) para resolverlo. Entre los algoritmos empleados destacan los Algoritmos Genéticos (Genetic Algorithms, GA), que se basan en la Teoría de Darwin de la evolución de las especies, y los algoritmos de Optimización por Cúmulo de Partículas (Particle Swarm Optimization, PSO), inspirados en el comportamiento social de los animales como las aves en una bandada (Blum y Roli, 2003).

Estos algoritmos inteligentes, combinados con un simulador de redes vehiculares realista, han calculado de forma automática configuraciones óptimas que han mejorado considerablemente el rendimiento de los protocolos de comunicación analizados.

Asimismo, hemos decidido sacar la experimentación del laboratorio y llevarla a la calle. Por lo que una vez mejorado los protocolos de comunicación, se han realizado pruebas de campo con vehículos equipados con dispositivos que faciliten la comunicación empleando una VANET circulando por las calles de Málaga. En estas pruebas sólo los protocolos optimizados con nuestra metodología han podido transmitir la información que se requería de un vehículo a otro con los requisitos establecidos.

Si revisamos la literatura, podemos encontrar trabajos que han aplicado diferentes metaheurísticas para la optimización de protocolos, ya sea para mejorar la calidad de servicio que ofrecen como para reducir los requisitos de recursos que necesitan (Bitam S. y Mellouk, 2014; Toutouh, 2016). La optimización de protocolos de difusión (broadcasting) para redes vehiculares fue analizada por Abdou y col. (2011) y GalavizMosqueda y col. (2016). Estos autores aplicaron algoritmos metaheurísticos basados en GA para mejorar el rendimiento de estos protocolos y reducir los problemas de congestión de la red. A su vez, se han aplicado este tipo de soluciones para optimizar protocolos de encaminamiento (routing). Lobiyal y col. (2015) aplicó un algoritmo inspirado en PSO para mejorar la entrega de paquetes de datos aplicando el protocolo de encaminamiento AODV. En Toutouh y Alba (2015), analizamos la optimización del mismo protocolo de enrutamiento aplicando dos algoritmos de optimización multiobjetivo, uno basado en GA y otro en PSO. Otro protocolo de encaminamiento que también se ha estudiado es el OLSR. Un conjunto de cinco metaheurísticas fueron aplicadas para optimizar dicho protocolo (Toutouh y col, 2012). En todos estos trabajos previos validan sus resultados empleando simuladores de VANET, lo que limita el realismo de los resultados que ofrecen. Nosotros por el contrario, en el presente trabajo lo hacemos empleando experimentos de campo. 


\section{WPSReview International on Sustainable \\ Housing and Urban Renewal (RI-SHUR)}

Así, los objetivos principales de este trabajo de investigación son los siguientes: a) introducir al lector a la revolucionaria tecnología de las redes vehiculares y la CSD; b) enumerar los principales retos que presenta su desarrollo, centrándose en la optimización de los protocolos; c) aplicar metaheurísticas para resolver dicho problema; y d) validar mediante pruebas reales de campo los resultados obtenidos.

Este documento se organiza como sigue. En la siguiente sección introducimos el concepto de VANET y discutimos sus principales problemas. La Sección 3 presenta el problema de optimización definido para mejorar el protocolo de transferencia de archivos. La Sección 4 describe la metodología empleada para resolver el problema. Las secciones 5 y 6 presentan los resultados experimentales de la optimización del protocolo y las pruebas de campo, respectivamente. Finalmente, en la Sección 7, se presentan las conclusiones de este trabajo y las principales líneas de trabajo futuro.

\section{2.- Comunicación Eficiente en Redes Vehiculares}

Uno de los pilares del CSD son las redes vehiculares ad hoc o VANET (vehicular ad hoc networks). Las VANET son redes descentralizadas que proveen de una plataforma para el diálogo contínuo de vehículos entre sí (vehicle-to-vehicle o V2V) y con los elementos de la infraestructura vial (vehicle-to-infrastructure o V2I) como semáforos, señales de tráfico, etc. Asimismo, aprovechando que los vehículos pueden contar con dispositivos de conexión a redes celulares (como teléfonos móviles) también se definen las comunicaciones de banda ancha (vehicle-to-broadband o V2B), aunque solo para cierto tipo de servicios (Hartenstein y Laberteaux, 2009; Campolo y col., 2015).

Figura 2. Escenario VANET típico

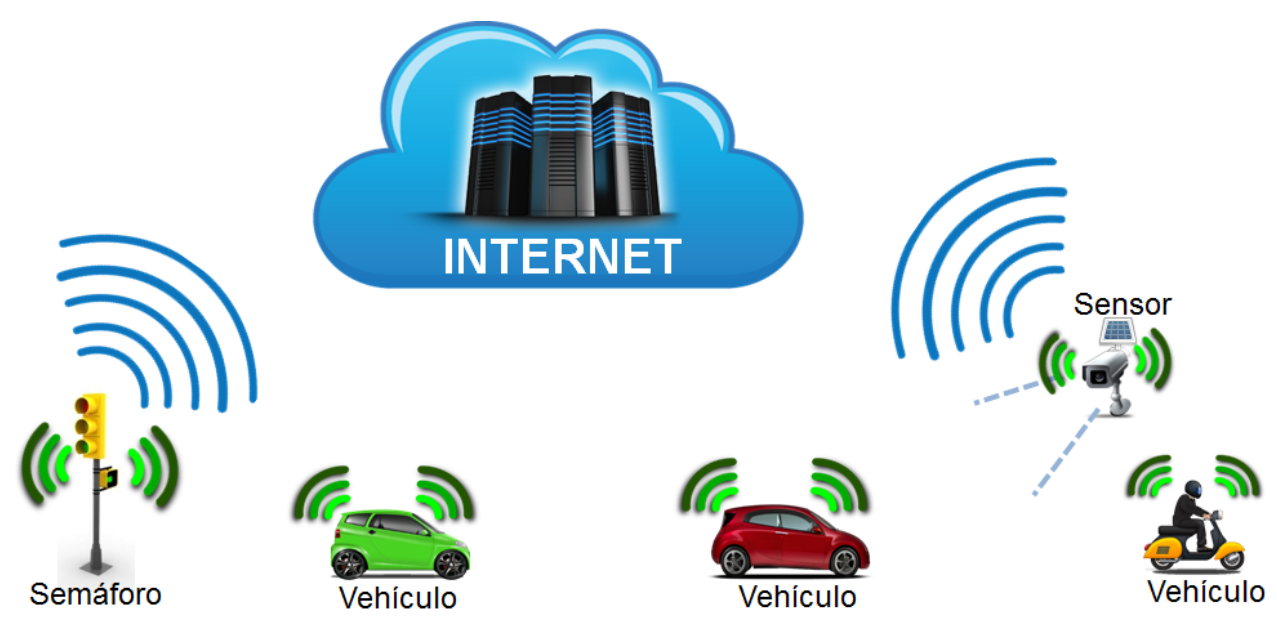

WPS RI-SHUR, no7-8, 2018, vol.1, ISSN: 2387-1768 


\title{
WPSReview International on Sustainable
}

\author{
Housing and Urban Renewal (RI-SHUR)
}

Debido a que las VANET comunican de forma directa y a que los vehículos están en continuo movimiento, cuentan con una serie de características que las hace únicas dentro del dominio de las redes móviles. Estas son las siguientes:

- Nodos con alta capacidad de cómputo y energía: Los vehículos que pertenecen a las VANET cuentan con ordenadores de abordo que tienen una capacidad de cómputo muy competitiva. A su vez, estos dispositivos se encuentran conectados a la fuente de energía del vehículo (batería y alternador) por lo que no tienen restricciones de energía elevada.

- Control totalmente descentralizado: No existe ninguna entidad central o servidor que se encargue de la gestión de la red, por lo que se hace de forma totalmente distribuida entre los nodos de la red.

- Alta movilidad, pero predecible de los nodos: La mayoría de los nodos de las VANET son vehículos que se mueven a altas velocidades. Asimismo, los vehículos se mueven siguiendo los patrones que les indican las carreteras y las normas de circulación, por lo que se puede predecir cuál va a ser el movimiento que lleve un nodo.

- Topología de la red altamente variable: La topología formada por los nodos de la red tiende a cambiar de forma rápida y continua. Esto se debe a principalmente dos factores que dificultan el mantenimiento de la conectividad entre nodos. Estos son: a) los nodos de las VANET emplean tecnologías de comunicación inalámbrica basadas en los protocolos de IEEE 802.11 (corto alcance) y $b$ ) la movilidad de los nodos es muy alta.

- Variabilidad en las condiciones en las que se da la comunicación: Las VANET se pueden desplegar en diferentes entornos, tanto en áreas urbanas como interurbanas. En las primeras la existencia de edificios, árboles y todo tipo de obstáculos limitan la propagación de la señal inalámbrica, y con ello, la calidad de servicio de la red. Fuera de las ciudades, las condiciones de propagación de la señal son más favorables, pero los nodos se mueven a mayor velocidad.

Estas características hacen del despliegue de este tipo especial de redes un desafío formidable, puesto que la mayoría de sus servicios requieren comunicaciones con unos umbrales de una calidad de servicio altos y estrictos en términos de tiempos y ratios de entrega. Por lo tanto, es necesario que los protocolos de comunicación que se empleen en VANET tengan un funcionamiento óptimo. Para así, el intercambio de información/archivos entre vehículos se haga de forma correcta y dentro de los tiempos requeridos. Esto es importante puesto que un paquete de datos o un archivo llegue en tiempo y forma puede ser la diferencia de evitar un accidente de tráfico o no.

Diferentes autores han trabajado principalmente en dos líneas de investigación para la obtención de protocolos útiles para comunicaciones vehiculares:

- Se han desarrollado un protocolo específico para el intercambio de archivos entre vehículos empleando comunicaciones VANET, el VDTP (Vehicular Data Transfer Protocol) (Luna S., 2008). Este protocolo permite a dos vehículos intercambiar archivos que contengan datos, imágenes, audio y vídeo. 


\title{
WPSReview International on Sustainable
}

\author{
Housing and Urban Renewal (RI-SHUR)
}

- Se ha definido una metodología para optimizar protocolos de comunicación para redes móviles ya existentes. Para que así, estos cumplan con las restricciones de calidad de servicio cuando se usan en entornos de alta volatilidad como las redes vehiculares.

Aquí nos centramos en la segunda línea de trabajo, la optimización de protocolos. La idea fundamental es la de encontrar una configuración de los parámetros que gobiernan el funcionamiento del protocolo (contadores, temporizadores, variables de decisión, etc.) que mejor se adapten a las características de las VANET, y así ofrezcan la mejor calidad de servicio posible. Se define un proceso de optimización que se encarga de buscar de forma automática e inteligente una configuración de parámetros que mejoren de forma significativa diferentes aspectos de la comunicación, como por ejemplo, tiempos de transmisión, tasas de transferencia, etc.

Los parámetros de configuración de un protocolo tienen una fuerte relación no-lineal entre ellos y tienen una influencia difícil de evaluar de forma directa en la calidad de servicio que ofrecen. Así, una configuración simboliza un conjunto de valores continuos y discretos que dificultan la búsqueda de una configuración óptima en entornos de comunicación reales empleando técnicas de optimización clásicas (Toutouh, 2016). Por lo tanto, es necesario aplicar métodos inteligentes en los que destacan las metaheurísticas, como los Algoritmos Genéticos y los algoritmos de Optimización por Cúmulo de Partículas, capaces de obtener soluciones (configuraciones de protocolos) eficientes empleando recursos de cómputo razonables. A esto lo llamamos optimización de protocolos off-line.

\section{3.- Problema de la Optimización de la Transferencia de Archivos en VANET}

El protocolo de transferencia de archivos VDTP fue desarrollado por miembros de nuestro grupo de investigación en el contexto del proyecto europeo EUREKA-CELTIC CARLINK (CARLINK, 2006). El protocolo opera en la capa de aplicación y se encarga de ofrecer un servicio transversal a todas las aplicaciones de CSD que se pueden desarrollar sobre una VANET, la transferencia de información entre dos vehículos encapsulada en archivos. Así, VDTP se emplea para transferir tanto los datos de sobre la cinemática del vehículo (velocidad, dirección, aceleración, etc.) empleados por aplicaciones de seguridad vial (archivos del orden de decenas de kilobytes), como archivos multimedia (audio o vídeo) que tienen tamaños del orden de decenas de megabytes.

VDTP es un protocolo basado en el método de parada y espera (stop-and-wait) específicamente diseñado para comunicaciones vehiculares (Luna S., 2008). El protocolo divide los archivos en segmentos, paquetes o tramas de un tamaño determinado y los envía de forma consecutiva, de modo que no se envía la trama siguiente hasta que no se recibe un paquete de confirmación de recepción (ACK) por parte del receptor del archivo. Así, el emisor espera un tiempo de espera determinado, si en ese tiempo no ha recibido el ACK reenvía la trama actual. Este proceso de espera y reenvío se realiza un número determinado de veces por trama, una vez

WPS RI-SHUR, no7-8, 2018, vol.1, ISSN: 2387-1768 


\section{WPSReview International on Sustainable \\ Housing and Urban Renewal (RI-SHUR)}

llegado a ese número de veces se entiende que la comunicación entre emisor y receptor se ha perdido y se detiene la transferencia.

Como se puede comprobar por el funcionamiento de VDTP, éste depende esencialmente de tres parámetros fundamentales que condicionan su funcionamiento y su rendimiento (Luna S., 2008). Estos son los siguientes:

- El tamaño de segmento que es un valor entero que representa el número de bytes de datos máximos que puede encapsular una trama. Según la definición del protocolo está en el rango de que va de 128 a 524.288 (512 kilobytes).

- El tiempo de espera para la retransmisión de una trama en segundos que es un valor real que va de 1 a 10.

- El máximo número de reenvíos por segmento que es un valor entero que va del 1 al 250.

El problema de Optimización de la Transferencia de Archivos (OTA) en VANET que se trata en este estudio consiste en buscar una configuración para los tres parámetros de VDTP para mejorar la calidad del servicio del protocolo y asegurar el correcto funcionamiento de la CSD. Esto significa que se quiere minimizar los tiempos de transferencia de los archivos y el número de tramas perdidas y maximizar la cantidad de datos transferidos correctamente. Para ello se empleará una estrategia de optimización de protocolos off-line.

\section{4.- Metodología}

La optimización de protocolos off-line consiste en la búsqueda automática de configuraciones factibles de los parámetros que gobiernan los protocolos, para optimizar su rendimiento. El número y el rango de valores de los parámetros que gobiernan el protocolo definen un espacio de búsqueda lo suficientemente grande y desconocido para hacer inútil el uso de métodos exactos y enumerativos para resolverlo.

La metodología de optimización seguida en este trabajo consiste en aplicar de forma conjunta una metaheurística y un proceso de simulación de una VANET que calcule la calidad de las soluciones tentativas (configuraciones del protocolo que se optimiza) que se van creando. Así, las diferentes soluciones que calculan los algoritmos de optimización son evaluadas por un simulador que configura los nodos con la solución a evaluar. Tras la simulación, se analizan distintas métricas sobre las comunicaciones, y con ellas se evalúa la calidad o fitness de la solución (ver Figura 2). 


\title{
WPSReview International on Sustainable
}

\author{
Housing and Urban Renewal (RI-SHUR)
}

Figura 2. Estrategia de optimización off-line de protocolos VANET

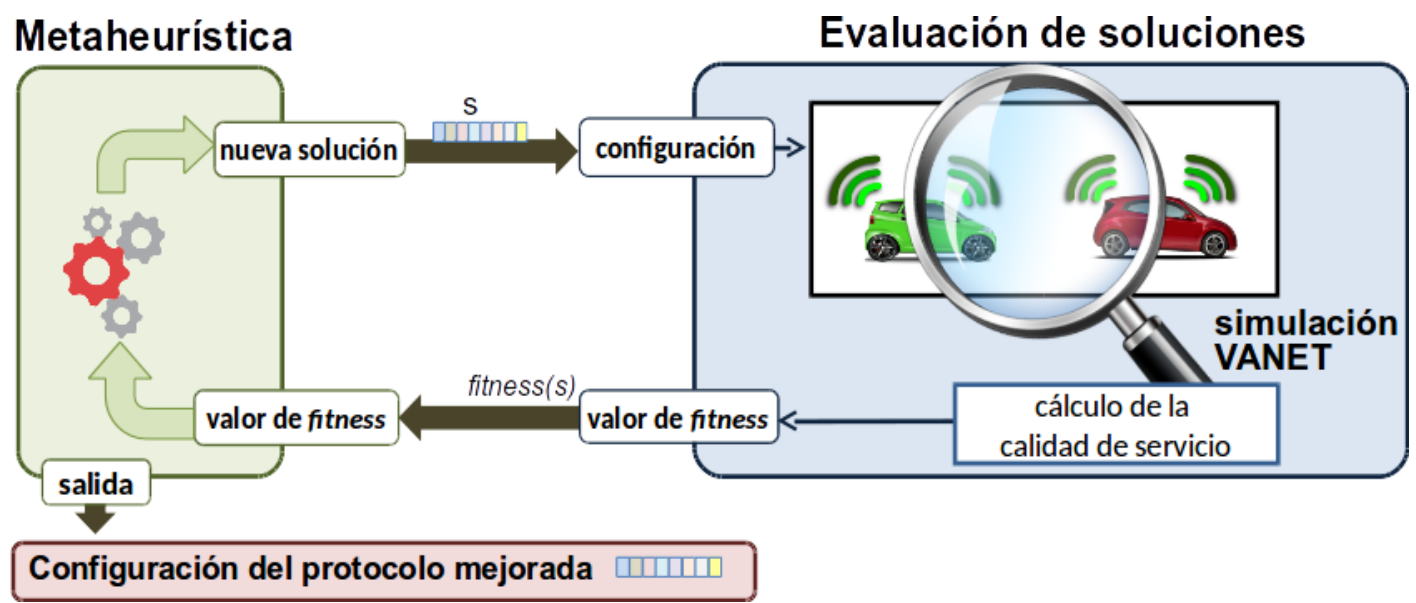

En este trabajo de investigación se presenta la optimización del protocolo de transferencia de archivos VDTP. La idea principal es la de maximizar la cantidad de datos que se envían dos vehículos. Para ello se ha evaluado la calidad del servicio que ofrece el protocolo en términos de cantidad de datos transmitidos en kilobytes (datos_transmitidos), número de segmentos de datos perdidos (segmentos_perdidos) y tiempo de transmisión de los archivos en segundos (tiempo_transmision), en un escenario en el que diferentes vehículos intercambian un número $N$ de archivos.

Para que las metaheurísticas empleadas en este trabajo puedan realizar la búsqueda de una configuración óptima se ha de definir una función de calidad o fitness, que tenga en cuenta los tres parámetros de calidad de servicio empleados en este estudio. La siguiente ecuación se ha definido de forma que el problema OTA se resuelva mediante la minimización de la función $f$ de fitness, y con ello, se maximice la cantidad de datos transmitidos y se minimice tanto el tiempo de transmisión como el número de segmentos perdidos.

$$
f=\frac{1}{N} \sum_{i=1}^{N} \frac{\text { tiempo_transmision }_{i} \text {-segmentos_perdidos }}{i}
$$

Para que el proceso de optimización obtenga una configuración óptima y aplicable al mundo real, la simulación debe obtener resultados fidedignos y realistas. Así, se han definido escenarios realistas empleando información real de la ciudad de Málaga para la evaluación de los protocolos en la simulación. Para ello se ha empleado un simulador utilizado por numerosos autores, el Network Simulator 2 o ns-2 (Issariyakul, 


\section{WPSReview International on Sustainable \\ Housing and Urban Renewal (RI-SHUR)}

T. y E. Hossain, 2008). Cabe destacar que el uso de este tipo de simulaciones requieren de unos tiempos de cómputo que están en el orden de minutos.

Con afán de asegurar que los resultados obtenidos son útiles para la sociedad, hemos decidido sacar la investigación del laboratorio a las calles. Así, hemos realizado pruebas reales transferencia de archivos entre vehículos empleando el protocolo VDTP, configurando el mismo con las parametrizaciones obtenidas de forma automática por nuestra metodología y la estándar propuesta por los diseñadores.

Cinco optimizadores basados en metaheurísticas se han analizado en este trabajo para resolver OTA (Blum y Roli, 2003): cuatro basadas en población o conjunto de soluciones (PSO, GA, Evolución Diferencial o Differential Evolution -DE- y Estrategias Evolutivas o Evolutionary Strategies -ES-) y una basada en trayectoria (Enfriamiento Simulado o Simulated Annealing -SA-).

\section{5.-Resultados y Conclusiones}

\section{Evaluación Experimental}

Esta sección describe los experimentos llevados a cabo para resolver el problema de optimización OTA empleando metaheurísticas. Los algoritmos utilizados para resolver dicho problema de optimización han sido implementados en $\mathrm{C}_{++}$empleando la biblioteca de funciones MALLBA desarrollada por nuestro grupo de investigación (Alba y col., 2006). Para la evaluación de las soluciones se ha empleado ns-2 en su versión 2.31. Como las metaheurísticas son algoritmos no deterministas, se realizan 30 ejecuciones independientes de cada una de las metaheurísticas.

\subsection{Instancia VANET para la Simulación de las Soluciones}

Para la evaluación (simulación) de las soluciones generadas por las metaheurísticas se ha definido un escenario a partir de los datos reales de una zona cercana al Campus de Teatinos de la Universidad de Málaga en Málaga. Se simula un tiempo de 180 segundos en los que hay 30 vehículos recorriendo las carreteras que se muestran en la Figura 3 siguiendo las normas reales de tráfico y tratando de intercambiando archivos de 1024 kilobytes entre ellos usando VDTP. Para que los resultados de la simulación reflejen las interacciones reales de las comunicaciones se han seguido las recomendaciones propuestas por Alba y col. (2008). 


\section{WPSReview International on Sustainable}

\section{Housing and Urban Renewal (RI-SHUR)}

Figura 3. Carreteras pertenecientes al escenario simulado para la evaluación de soluciones.

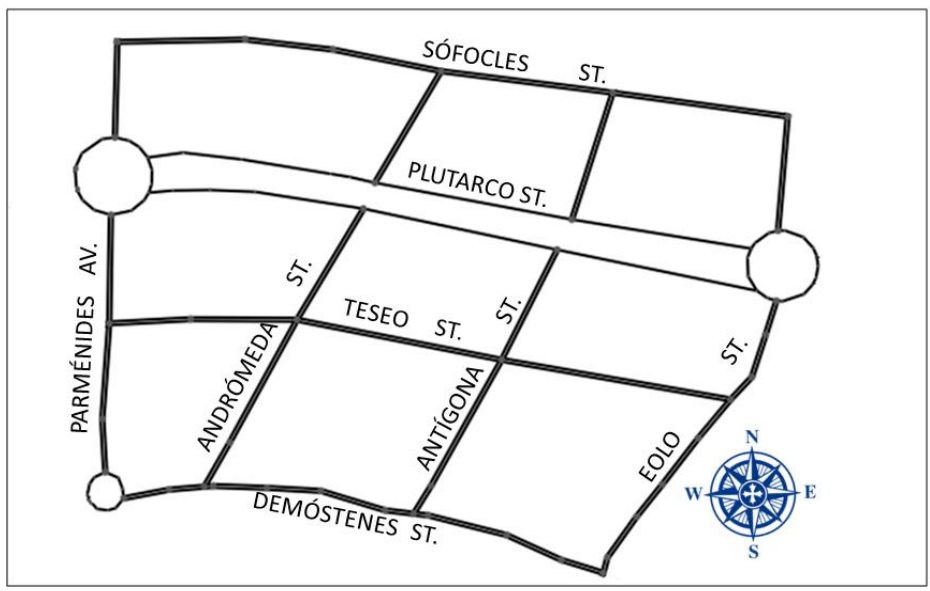

\subsection{Configuración de los Algoritmos}

Para que la comparativa entre algoritmos sea justa estos se han configurado teniendo en cuenta que todos realicen el mismo número de máximo soluciones tentativas, es decir, que el criterio de parada de los cinco algoritmos será que lleven a cabo 1000 evaluaciones de la función de fitness. Cada evaluación de una solución invoca al simulador ns-2 que tras simular la misma devuelve los valores de las tres métricas que tiene en cuenta la función del fintness. Es importante que cada simulación requiere del orden de minutos para ejecutarse.

Para que todos los algoritmos ejecuten 1000 evaluaciones del fitness se han configurado los algoritmos basados en población (PSO, DE, GA y ES) con una población de 20 individuos y 50 generaciones. EI SA se ha configurado para que lleve 1000 iteraciones, puesto que trabaja con una única solución. Los demás parámetros de cada algoritmo se han configurado como se muestra en la Tabla 1.

Tabla 1. Configuración de las metaheurísticas para resolver OTA.

\begin{tabular}{|l|l|l|}
\hline Algoritmo & Parámetro & Valor \\
\hline \multirow{3}{*}{ PSO } & Coeficiente local & 2.0 \\
& Coeficiente social & 2.0 \\
& Peso de inercia & 0.5 \\
\hline
\end{tabular}

WPS RI-SHUR, no7-8, 2018, vol.1, ISSN: 2387-1768 
WPSReview International on Sustainable

Housing and Urban Renewal (RI-SHUR)

\begin{tabular}{|l|l|l|}
\hline DE & Probabilidad de cruce & 0.9 \\
& Probabilidad de mutación & 0.1 \\
\hline \multirow{2}{*}{ GA } & Probabilidad de cruce & 0.8 \\
& Probabilidad de mutación & 0.2 \\
\hline \multirow{2}{*}{ ES } & Probabilidad de cruce & 0.9 \\
& Probabilidad de mutación & 0.1 \\
\hline SA & Bajada de temperatura & 0.8 \\
\hline
\end{tabular}

\subsection{Resultados numéricos}

La Tabla 2 resume los resultados obtenidos al aplicar las cinco metaheurísticas para resolver el problema de OTA sobre el escenario que se muestra en la Sección 5.1. Los resultados se muestran en términos de media y desviación estándar normalizada, mínimo (mejor fitness), mediana y máximo valores de fitness calculados en las 30 ejecuciones independientes.

Tabla 2. Resultados numéricos en términos de valores final de fitness.

\begin{tabular}{|l|l|l|l|l|}
\hline Algoritmo & Media \pm Desv. & Mínimo & Mediana & Máximo \\
\hline PSO & $\mathbf{1 . 6 3 4 6} \pm 17.74 \%$ & 0.9077 & $\mathbf{1 . 7 8 0 9}$ & $\mathbf{1 . 8 9 1 8}$ \\
\hline DE & $1.7423 \pm 21.33 \%$ & $\mathbf{0 . 7 3 8 9}$ & 1.8658 & 2.0228 \\
\hline GA & $1.9086 \pm 11.84 \%$ & 0.8799 & 1.9731 & 2.1614 \\
\hline ES & $2.1517 \pm 5.88 \%$ & 1.8862 & 2.1222 & 2.4246 \\
\hline SA & $2.7850 \pm 31.30 \%$ & 0.8730 & 2.1663 & 3.8025 \\
\hline
\end{tabular}

PSO es la metaheurística que obtiene los mejores resultados en términos de media, mediana y máximo valores de fitness. Estos resultados nos llevan a asumir que las 


\section{WPSReview International on Sustainable}

\section{Housing and Urban Renewal (RI-SHUR)}

configuraciones de VDTP obtenidas empleando PSO ofrecen una transferencia de archivos rápida y robusta. Sin embargo, cabe destacar que la mejor de las configuraciones (mínimo fitness) ha sido calculada por DE.

Para proveer de una comparativa con significancia estadística, se ha aplicado el Test de Friedman con una significancia del 99\% (Sheskin, 2007) a la distribución de los resultados para obtener un ranking. Así, el test ha devuelto que existe una diferencia significativa entre los resultados y que PSO es el algoritmo más competitivo de los cinco analizados. El ranking se completa con DE, GA, ES y SA, como segundo, tercero, cuarto y quinto respectivamente. Con estos resultados se puede concluir que PSO es la metaheurística que ha obtenido mejores resultados resolviendo OTA.

\subsection{Análisis de la Calidad de Servicio de las Soluciones}

Para analizar las soluciones (configuraciones de VDTP) obtenidas empleando la metodología que se ha utilizado en este trabajo, hemos realizado una comparativa de las mejores configuraciones de cada una de las metaheurísticas con las que propusieron los expertos del proyecto CARLINK. Las configuraciones analizadas y los resultados se muestran en la Tabla 3. Para ello hemos vuelto a simular dichas configuraciones sobre el escenario presentado en la Sección 5.1. La Tabla 3 también presenta los resultados.

Tabla 3. Resultados numéricos en términos de valores final de fitness.

\begin{tabular}{|l|l|l|l|l|l|l|}
\hline \multirow{2}{*}{ Algoritmo } & \multicolumn{3}{|l|}{ Configuración VDTP } & \multicolumn{3}{l|}{ Resultado de la simulación } \\
\cline { 2 - 8 } & $\begin{array}{l}\text { Tamaño } \\
\text { de } \\
\text { segmento }\end{array}$ & $\begin{array}{l}\text { Tiempo } \\
\text { de } \\
\text { espera }\end{array}$ & $\begin{array}{l}\text { Max. num. } \\
\text { repeticiones }\end{array}$ & $\begin{array}{l}\text { Tiempo de } \\
\text { transmisión }\end{array}$ & $\begin{array}{l}\text { Paquetes } \\
\text { perdidos }\end{array}$ & $\begin{array}{l}\text { Datos } \\
\text { transmitidos }\end{array}$ \\
\hline PSO & 41358 & 10,00 & 3 & 3,41 & 0,27 & 1024 \\
\hline DE & 28278 & 6,00 & 9 & 3,59 & 0,63 & 1024 \\
\hline GA & 31196 & 3,83 & 9 & 3,61 & 0,27 & 1024 \\
\hline ES & 23433 & 10,00 & 8 & 3,50 & 0,27 & 1024 \\
\hline SA & 19756 & 6,43 & 3 & 4,22 & 0,36 & 1024 \\
\hline CARLINK & 25600 & 8,00 & 8 & 4,24 & 1,60 & 1024 \\
\hline
\end{tabular}

WPS RI-SHUR, no7-8, 2018, vol.1, ISSN: 2387-1768 


\section{WPSReview International on Sustainable \\ Housing and Urban Renewal (RI-SHUR)}

Los resultados que muestran la Tabla 3 muestran que todas las configuraciones simuladas han sido capaces de transferir de forma completa los archivos de 1024 kilobytes. Además, todas las configuraciones obtenidas de forma automática empleando metaheurísticas, mejoran la calidad del servicio que la configuración propuesta por los expertos de CARLINK. Se confirma que PSO es la metaheurística que mejores configuraciones para VDTP ha obtenido. Concretamente, la configuración de VDTP de PSO simulada ha transferido los datos en el menor tiempo posible $(3,41$ segundos), lo que representa una reducción de un 19,5\% del tiempo que requiere la configuración de los expertos. Asimismo, esta configuración es la que ha perdido el menor número de paquetes $(0,27)$.

Estos resultados se pueden traducir en términos de velocidad de transmisión de datos efectiva de la red (kilobytes por segundo). La Figura 4 muestra dichos resultados y se puede comprobar que para casi todos los casos, las configuraciones obtenidas de forma automática consiguen velocidades significativamente superiores.

Figura 4. Resultados en términos de velocidad de transmisión efectiva.

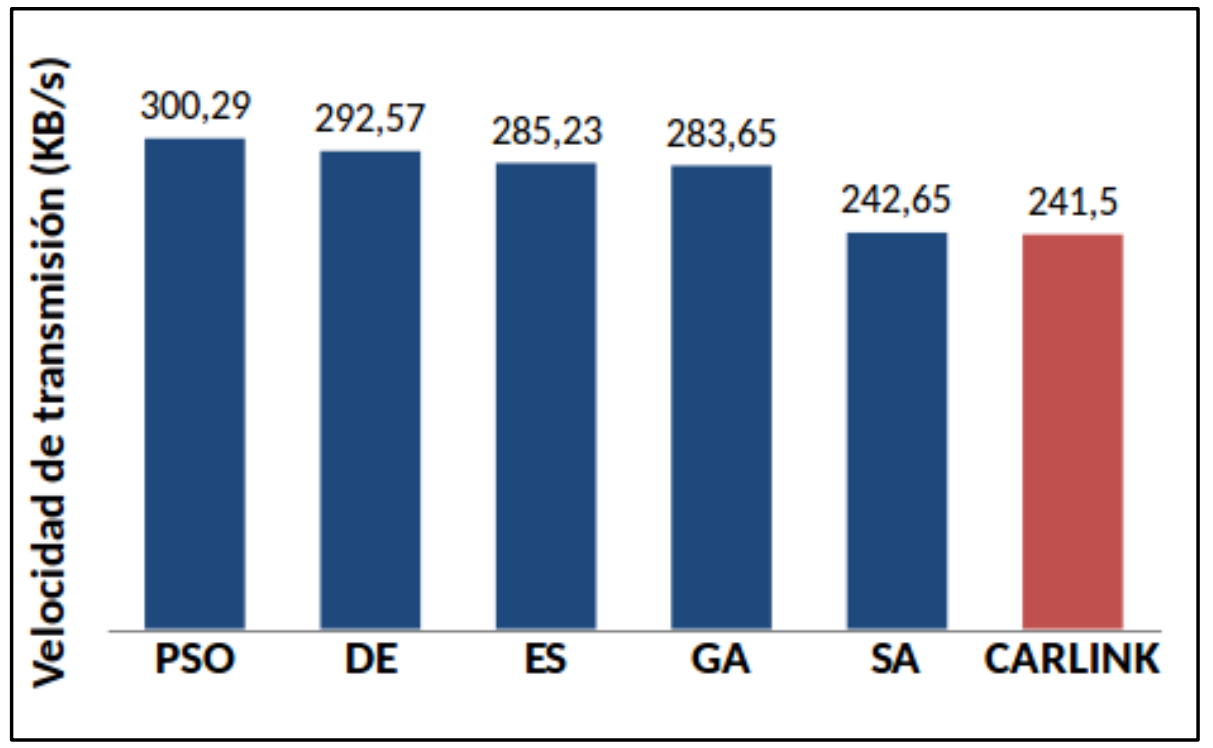

2. Validación de los Resultados en Pruebas de Campo

Para poder evaluar la validez de los resultados obtenidos mediante simulación sobre la optimización de protocolos, se ha llevado a cabo un estudio experimental empleando vehículos y dispositivos reales en las carreteras abiertas al tráfico de Málaga. 


\section{WPSReview International on Sustainable \\ Housing and Urban Renewal (RI-SHUR)}

La eficiencia de las configuraciones optimizadas frente a la propuesta por los expertos de CARLINK se ha analizado realizando transferencias de de archivos entre dos vehículos equipados tal y como se muestra en la Figura 5. Para cubrir una mayor variedad de servicios para CSD. Se han realizado la transferencia de archivos de distintos tamaños (100 KBytes, 500 KBytes, 1 MByte, 5 MBytes y 10 MBytes). A su vez se han evaluado las transferencias de archivos a distintas velocidades: primero, velocidades comprendidas entre 20 y $30 \mathrm{~km} / \mathrm{h}$ (velocidad reducida), y segundo, de entre 40 y $50 \mathrm{~km} / \mathrm{h}$ (velocidad moderada).

Figura 5. Equipamiento para realizar las pruebas de campo de CSD.

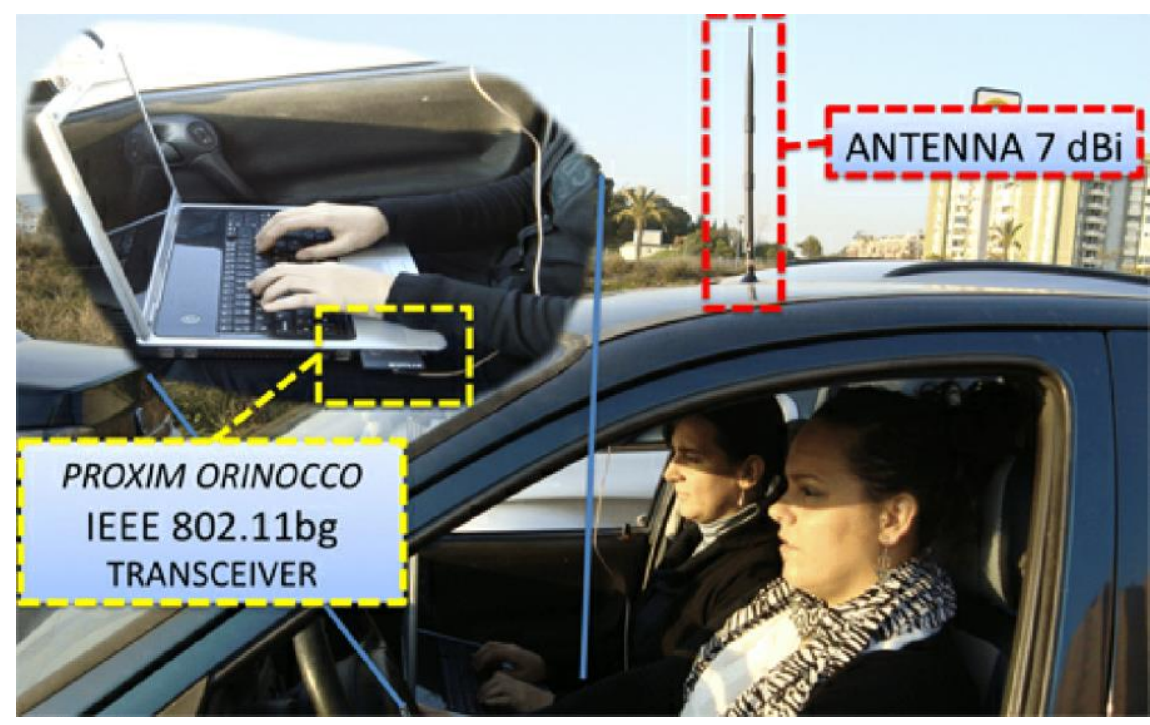

Como se puede observar en la Figura 6, durante las pruebas de campo los vehículos han estado a diferentes distancias y han tenido obstáculos entre sí. Esto mejora el realismo de los resultados que se obtienen, puesto que refleja una situación real donde se va a dar el CSD. 


\section{WPSReview International on Sustainable}

\section{Housing and Urban Renewal (RI-SHUR)}

Figura 6. Vehículos circulando durante las pruebas de campo.

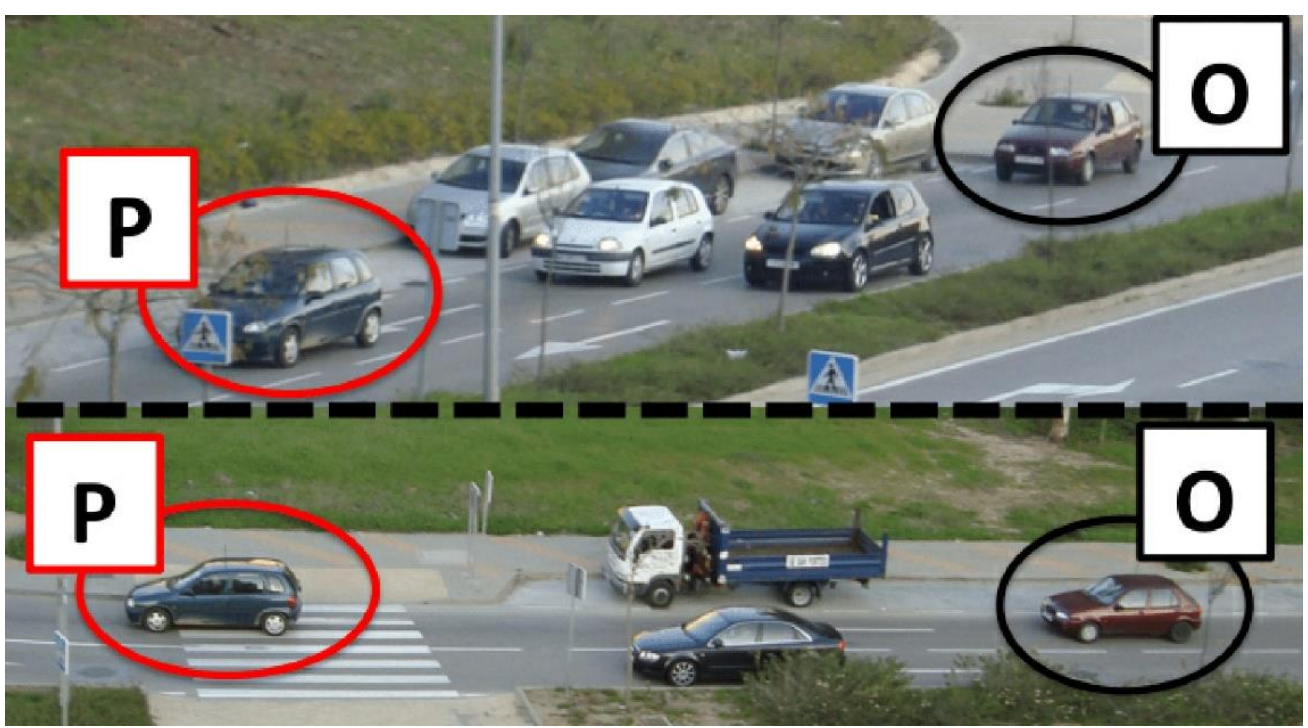

Los resultados se han analizado en términos de velocidad o tasa efectiva de transferencia (en kilobytes por segundo). De acuerdo con los resultados que se muestran en la Figura 7 se pueden sacar las siguientes conclusiones: a) los protocolos optimizados ofrecen una mayor velocidad de transferencia de datos que el estándar, b) a mayor tamaño del archivo que se transfiere, mayor es la tasa de transferencia de datos, y c) la velocidad afecta negativamente a la calidad de las comunicaciones. 
WPSReview International on Sustainable

Housing and Urban Renewal (RI-SHUR)

Figura 7. Resultados de las pruebas de campo.
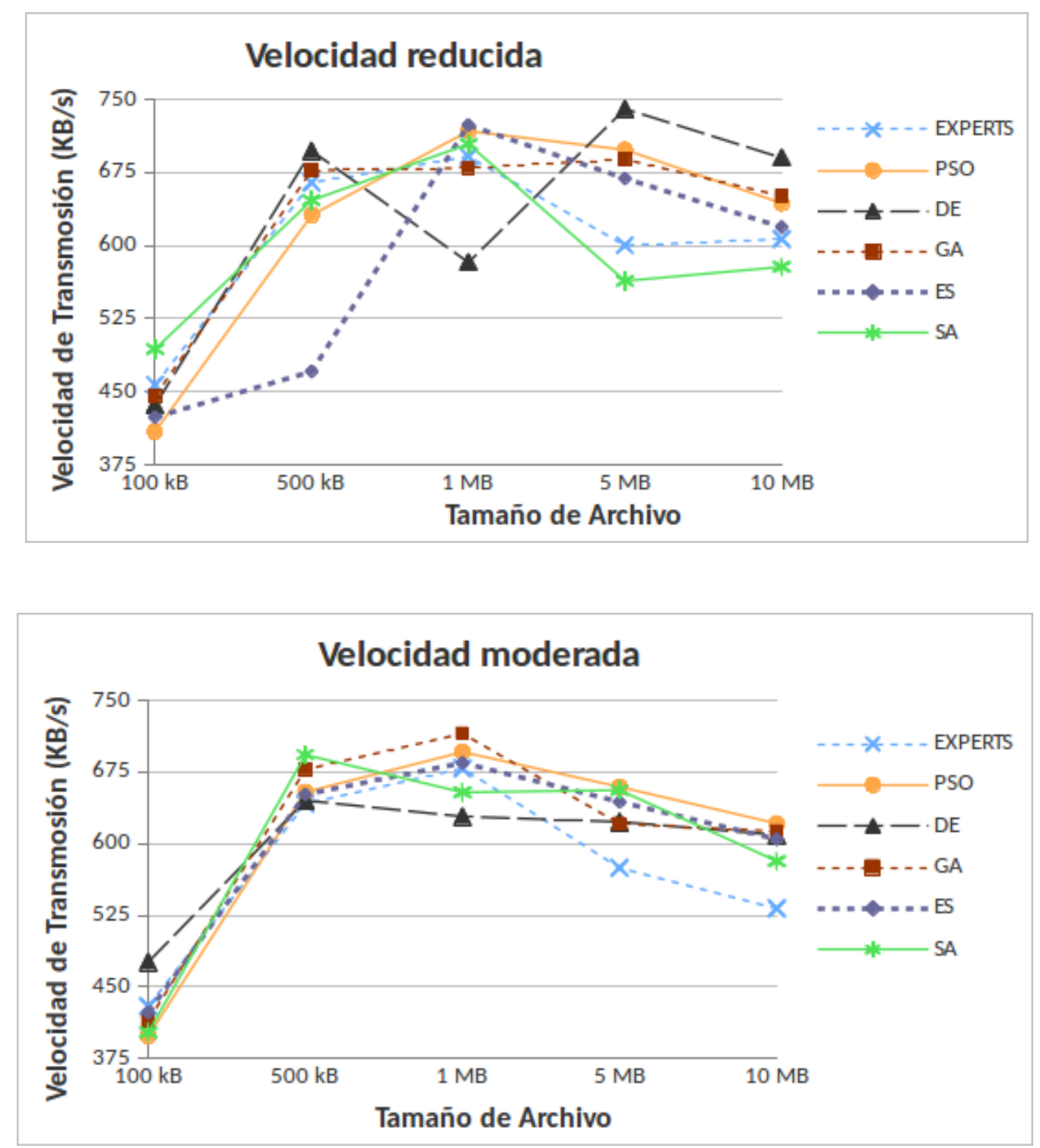

3. Conclusiones y Trabajo Futuro

En este trabajo se ha introducido la importancia de las redes vehiculares y la CSD en el diseño de soluciones innovadoras de movilidad inteligente. Así mismo, se han descrito los principales retos que presenta el despliegue de este tipo de tecnologías. Finalmente, nos hemos centrado en la optimización de protocolos, puesto que es fundamental para que las comunicaciones aseguren el buen funcionamiento de las aplicaciones basadas en CSD.

WPS RI-SHUR, no7-8, 2018, vol.1, ISSN: 2387-1768 


\section{WPSReview International on Sustainable \\ Housing and Urban Renewal (RI-SHUR)}

Se ha propuesto una metodología que optimiza protocolos obteniendo de forma inteligente y automática configuraciones de sus parámetros que se adaptan mejor a los requisitos de las VANET. Específicamente, se ha tratado la optimización del protocolo VDTP de transferencia de archivos mediante la definición del problema de optimización OTA. Este problema se ha tratado aplicando cinco metaheurísticas (PSO, DE, GA, ES y SA). PSO ha obtenido los resultados más competitivos en la resolución de OTA.

Asimismo, se han realizado pruebas reales de campo para comprobar el buen funcionamiento y la mejora de las configuraciones obtenidas de forma automática frente a la configuración propuesta por los expertos. Confirmándose que las configuraciones óptimas se comportan mejor que la que se propuso por los expertos de CARLINK.

Como trabajo futuro se barajan varias líneas: a) desarrollar aplicaciones reales de CSD a partir de VDTP, b) ampliar el conjunto de pruebas reales incluyendo un mayor número de vehículos y c) estudiar la aplicación de otras metaheurísticas para resolver otros problemas de optimización en movilidad inteligente (como por ejemplo, la selección de rutas en función de la información del tráfico, el diseño inteligente de la infraestructura vial, la configuración eficiente de los planes de los semáforos, etc.).

\section{6.- Referencias bibliográficas}

Abdou, W., A. Henriet, C. Bloch, D. Dhoutaut, D. Charlet, and F. Spies (2011). "Using an evolutionary algorithm to optimize the broadcasting methods in mobile ad hoc networks". In: Journal of Network and Computer Applications 34.6. Control and Optimization over Wireless Networks, pp. $1794-1804$.

Alba, E., F. Almeida, M. Blesa, C. Cotta, M. Diaz, I. Dorta, J. Gabarró, J. González, C. León, L. Moreno, J. Petit, J. Roda, A. Rojas, y F. Xhafa (2006). "MALLBA: A library of skeletons for combinatorial optimisation". In: Parallel Computing 32.5-6, pp. 415-440.

Alba, E., S. Luna, and J. Toutouh (2008). "Accuracy and Efficiency in Simulating VANETs". In: Modelling, Computation and Optimization in Information Systems and Management Sciences, Second International Conference (MCO). Vol. 14. Communications in Computer and Information Science. Metz, France - Luxembourg: Springer, pp. 568-578.

Bitam S. y Mellouk A (2014). Bio-Inspired Routing Protocols for Vehicular Ad Hoc Networks. Wiley, Hoboken, NJ, USA, 2014.

Blum, C. y A. Roli (2003). "Metaheuristics in combinatorial optimization: Overview and conceptual comparison". In: ACM Computing Surveys (CSUR) 35.3, pp. 268-308.

C2C-CC (2015). CAR 2 CAR Communication Consortium (C2C-CC). [online] Disponible en: https:/www.car-2-car.org. Consultado en 2017. 


\section{WPSReview International on Sustainable \\ Housing and Urban Renewal (RI-SHUR)}

Campolo, C., A. Molinaro, y R. Scopigno, eds. (2015). Vehicular ad hoc Networks Standards, Solutions, and Research. Springer.

CARLINK (2006). CARLINK Label CP3-005. [online] Available in http://carlink.lcc.uma.es/. Consultado en 2018.

Galaviz-Mosqueda, A., Villarreal-Reyes, S., Galeana-Zapien, H., Rubio-Loyola, J., y R. Rivera-Rodriguez (2016). "Genetic tuning of fuzzy rule-based systems for multi-hop broadcast protocols for VANETs”. Telecommunication Systems, 63(3), 399-420.

Hartenstein, H. y K. Laberteaux (2009). VANET Vehicular Applications and InterNetworking Technologies. Intelligent Transport Systems. Upper Saddle River, NJ, USA: John Wiley \& Sons.

Issariyakul, T. y E. Hossain (2008). Introduction to Network Simulator NS2. 1st ed. Springer Publishing Company, Incorporated.

Lobiyal, D. K., Katti, C. P. y A. K. Giri (2015). "Parameter value optimization of ad-hoc on demand multipath distance vector routing using particle swarm optimization". Procedia Computer Science, 46, 151-158.

Luna S. (2008). "VDTP: A File Transfer Protocol for Vehicular Ad hoc Networks". Tech. rep. [Online]. Disponible en: http://neo.lcc.uma.es/staff/jamal/downloads/VDTP.pdf. CARINK.

Sheskin, D. J. (2007). Handbook of Parametric and Nonparametric Statistical Procedures. Chapman \& Hall/CRC.

Toutouh J (2016). Natural Computing for Vehicular Networks. PhD thesis, ETSI Informática, University of Malaga.

Toutouh, J., J. García-Nieto, and E. Alba (2012). "Intelligent OLSR Routing Protocol Optimization for VANETs". In: Vehicular Technology, IEEE Transactions on 61.4, pp. 1884-1894.

Toutouh J. y E. Alba (2015). "Parallel multi-objective metaheuristics for smart communications in vehicular networks". In: Soft Computing. In Press., pp. 1-13. 